\title{
RINGS WHOSE RIGHT MODULES ARE DIRECT SUMS OF INDECOMPOSABLE MODULES
}

\author{
BIRGE ZIMMERMANN-HUISGEN
}

\begin{abstract}
It is shown that, given a module $M$ over a ring with 1 , every direct product of copies of $M$ is a direct sum of modules with local endomorphism rings if and only if every direct sum of copies of $M$ is algebraically compact. As a consequence, the rings whose right modules are direct sums of indecomposable modules coincide with those whose right modules are direct sums of finitely generated modules.
\end{abstract}

1. Introduction and notation. It is well known that the rings whose right modules are direct sums of finitely generated modules are characterized by the fact that their pure exact sequences of right modules split (Gruson and Jensen [9]). According to Auslander [3], Ringel and Tachikawa [10], and Fuller and Reiten [7], the rings enjoying this property on both sides are precisely the rings of finite representation type. Moreover, as was shown by Chase [4], if a ring $R$ has the condition on the right, then every right $R$-module possesses an indecomposable decomposition $\left(R_{R}\right.$ being artinian). For $R$ commutative, the converse was established by Warfield [13] who left the general case open. From our main theorem (Theorem A) it will follow that Warfield's result remains true for arbitrary rings. Evidence that this might be true has recently been provided by Fuller [8] who proved that the rings whose right modules are direct sums of finitely generated modules coincide with those whose right modules have decompositions that complement direct summands in the sense of [1]. Theorem $A$ also furnishes a more general background for this result.

An intermediate step in the proof of Theorem A deserves emphasis: Every $\Sigma$-algebraically compact module $M$ (i.e. all direct sums of copies of $M$ are algebraically compact) has the exchange property which in turn implies a certain cancellation property for $M$ (Theorem B).

Throughout, $R$ is an associative ring with identity and $R$-module means unitary right $R$-module. Recall that $M_{R}$ is algebraically compact (abbreviated by a.c. in proofs) if each finitely solvable system of equations $\sum_{i \in I} X_{i} a_{i j}=m_{j}$ $(j \in J)$, where $\left(a_{i j}\right)_{i \in I_{j} \in J}$ is a column-finite $R$-matrix and $m_{j} \in M$, is solvable (finitely solvable means that for each finite subset $J^{\prime}$ of $J$ there is an element $\left(n_{i}\right) \in M^{I}$ with $\sum_{i \in I} n_{i} a_{i j}=m_{j}$ for $\left.j \in J^{\prime}\right)$. More manageable char-

Received by the editors February 24, 1978 and, in revised form, October 5, 1978.

AMS (MOS) subject classifications (1970). Primary 16A46, 16A64; Secondary 16A52.

Key words and phrases. Indecomposable module, ring of finite representation type, exchange property, cancellation property. 
acterizations of $(\Sigma$-)algebraically compact modules and large classes of examples can be found in [14], [15]. We will make constant use of Warfield's observation that algebraically compact $=$ pure-injective [11, Theorem 2], as well as of the fact that the endomorphism ring of an indecomposable algebraically compact module is local [15, Theorem 9].

The author would like to express her gratitude to Professor C. U. Jensen for a stimulating conversation in which he conjectured a modified version of $(1) \Rightarrow(3)$ of Theorem A.

\section{Theorems.}

THEOREM A. For an $R$-module $M$ the following statements are equivalent:

(1) The countably infinite product $M^{\mathbf{N}}$ of copies of $M$ is a direct sum of submodules with local endomorphism rings.

(2) Every (pure submodule of a) direct product of copies of $M$ has a decomposition that complements direct summands.

(3) $M$ is $\Sigma$-algebraically compact.

Before giving a proof of Theorem A, we point out several consequences.

Corollary 1. Suppose that $M_{R}$ is algebraically compact (resp., injective). Then every (countable) direct product of copies of $M$ is a direct sum of indecomposable modules iff $M$ is $\Sigma$-algebraically compact (resp., $\Sigma$-injective).

Note that the second statement includes the well-known theorem of Matlis-Papp, see [6].

Proof. In view of Theorem $A$ the first claim follows from the fact that direct products of a.c. modules are again a.c., and indecomposable a.c. modules have local endomorphism rings. The second is a special case of the first.

COROLlARY 2. The following statements about a ring $R$ are equivalent:

(1) Every (algebraically compact) right $R$-module is a direct sum of indecomposable modules.

(2) Every right $R$-module has a decomposition that complements direct summands.

(3) All pure inclusions of right $R$-modules split.

Proof. (3) $\Rightarrow(2)$. Since all pure inclusions split, all right $R$-modules are $(\Sigma$-)a.c. Apply Theorem A. $(2) \Rightarrow(1)$ is clear, since the decompositions in (2) are necessarily indecomposable. (1) $\Rightarrow(3)$. As is well known [11, Corollary 6], each module $M_{R}$ can be embedded as a pure submodule into an a.c. module which by hypothesis and Corollary 1 is even $\Sigma$-a.c. But since $\Sigma$-algebraic compactness is inherited by pure submodules (see the criterion preceding Lemma 4), $M$ is in turn algebraically compact, which implies (3).

RemarK. As mentioned above, $(2) \Leftrightarrow(3)$ of Corollary 2 is known. Apart from Chase's contribution, the proof given here is completely different from 
Fuller's proof [8]; in particular, it does not use Harada's results on rings with enough idempotents.

As was discovered by Anderson and Fuller [1, Theorems 5, 6], right perfect (resp., semiperfect) rings are characterized by the fact that their projective (resp., finitely generated projective) right modules have decompositions that complement direct summands. We pursue this line of results in the following specialized version of Theorem $A$.

COROLlaRY 3. For a ring $R$ the following statements are equivalent:

(1) Every pure submodule of a direct product of copies of $R_{R}$ has a decomposition that complements direct summands.

(2) $R_{R}^{N}$ has a decomposition that complements direct summands.

(3) $R_{R}$ is $\Sigma$-algebraically compact.

REMARK. A right $\Sigma$-a.c. ring $R$ need not be left coherent; in particular, the products considered in Corollary 3 need not be projective. For typical examples of $\Sigma$-a.c. rings see [15].

An important tool in the proof of $(1) \Rightarrow(3)$ of Theorem $A$ is the following adaptation of Chase's [5, Theorem 1.2]. To his ideas we only add the concept of a $p$-functor of $\operatorname{Mod} R$. This is a subfunctor of the forgetful functor $\operatorname{Mod} R \rightarrow \operatorname{Mod} Z$ that commutes with direct products, see [14]. Such a functor automatically commutes with direct sums. The $p$-functorial subgroups of a module $M_{R}$ include several important types of subgroups: All subgroups $M a$, where $a$ is a finitely generated left ideal of $R$, all annihilators in $M$ of subsets of $R$ and all finitely generated $\operatorname{End}\left(M_{R}\right)$-submodules of $M$, more generally, all subgroups $\operatorname{Hom}_{R}(A, M)(a)$ where $A$ is an $R$-module and $a \in A$.

By [14, 3.4], $M_{R}$ is $\Sigma$-algebraically compact iff $M$ satisfies the minimum condition on subgroups $P M$ where $P$ is a (pure left exact) $p$-functor.

LemMA 4. Suppose that the direct product $\Pi_{i \in \mathbf{N}} M_{i}$ of a family $\left(M_{i}\right)_{i \in \mathbf{N}}$ of $R$-modules is a direct sum of submodules $Q_{l}, l \in L$. Then, given a descending chain $P_{1} \supset P_{2} \supset P_{3} \supset \cdots$ of $p$-functors, there exist a natural number $n_{0}$ and a finite subset $L^{\prime}$ of $L$ such that

$$
q_{l}\left(P_{n_{0}} \prod_{i>n_{0}} M_{i}\right) \subset \bigcap_{n \in \mathbf{N}} P_{n} Q_{l}
$$

for all $l \in L \backslash L^{\prime}\left(q_{l}: \bigoplus_{k \in L} Q_{k} \rightarrow Q_{l}\right.$ is the natural projection).

We sketch the proof, since Chase's argument (see [4, Theorem 3.1] and [5, Theorem 1.2]) is slightly clarified by the use of $p$-functors.

Proof. Assume the conclusion to be false. Then a standard induction yields a sequence $\left(n_{i}\right)_{i \in \mathrm{N}}$ of natural numbers with $n_{i+1}>n_{i}$ and sequences of pairwise different elements $l_{i} \in L$ resp., $m_{i} \in P_{n_{i}}\left(\Pi_{j>n_{i}} M_{j}\right)$ so that

(1) $q_{l_{i}}\left(m_{i}\right) \notin P_{n_{i+1}} Q_{l_{i}}$, and

(2) $q_{l_{i}}\left(m_{j}\right)=0$ for $j<i$. 
Note that $m=\sum_{i \in \mathbf{N}} m_{i} \in \Pi_{j \in N} M_{j}$ is well defined (since the sums of the components are finite in view of $m_{i} \in \Pi_{j>n_{i}} M_{j}$ ); and that for $k \in \mathbf{N}$ we have

$$
q_{l_{k}}(m)=q_{l_{k}}\left(m_{k}\right)+q_{l_{k}}\left(\sum_{i>k} m_{i}\right) \neq 0,
$$

since the first summand does not lie in $P_{n_{k+1}} Q_{l_{k}}$ whereas the second does. But this contradicts $m \in \bigoplus_{l \in L} Q_{l}$.

The next lemma is considerably stronger than is required for the proof of Theorem A.

LeMma 5. If $\Pi_{i \in I} M_{i}$ is a direct sum of submodules with local endomorphism rings and if $P_{1} \supset P_{2} \supset P_{3} \supset \cdots$ is a chain of p-functors of Mod $R$, there is a natural number $n_{0}$ such that for each family $\left(A_{i}\right)_{i \in I}$ of indecomposable direct summands $A_{i}$ of $M_{i}$ we have $P_{n} A_{i}=P_{n_{0}} A_{i}$ for $n \geqslant n_{0}$ and almost all $i \in I$. In particular, each summand $A_{j}$ whose isomorphism type occurs an infinite number of times in the family $\left(A_{i}\right)_{i \in I}$ is $\Sigma$-algebraically compact.

Proof. First, suppose that $I$ is countable and write $I=\mathbf{N}$ for simplicity. Let $M=\prod_{i \in \mathrm{N}} M_{i}=\bigoplus_{l \in L} Q_{l}$ be a decomposition as in the hypothesis. Choose $n_{0} \in \mathbf{N}$ and a finite subset $L^{\prime}$ of $L$ according to Lemma 4, and for $m>\left|L^{\prime}\right|$ let $i_{1}, \ldots, i_{m} \geqslant n_{0}$ be pairwise different natural numbers. Since each $A_{i}$ has in turn a local endomorphism ring, the finite $\operatorname{sum} A_{i_{1}} \oplus \cdots \oplus A_{i_{m}}$ has the exchange property by [12, Proposition 1], hence there are pairwise different elements $l_{1}, \ldots, l_{m}$ of $L$ with

$$
M=\bigoplus_{k=1}^{m} A_{i_{k}} \oplus \underset{l \in L \backslash\left\{l_{1}, \ldots, l_{m}\right\}}{\bigoplus} Q_{l}
$$

or, equivalently, the canonical projection $q: M=\bigoplus_{l \in L} Q_{l} \rightarrow \bigoplus_{k=1}^{m} Q_{l_{k}}$ induces an isomorphism $\bigoplus_{k=1}^{\mathrm{m}} A_{i_{k}} \rightarrow \bigoplus_{k=1}^{\mathrm{m}} Q_{l_{k}}$. In order to show that $\left(P_{n}\right)_{n>n_{0}}$ is stationary on at least one of the $A_{i_{k}}$ 's let $l \in\left\{l_{1}, \ldots, l_{m}\right\} \backslash L^{\prime}$ (such an $l$ exists, because $m>\left|L^{\prime}\right|$ ). Adopting the notation of Lemma 4, we have

$$
P_{n_{0}} Q_{l}=q_{l}\left(\bigoplus_{k=1}^{m} P_{n_{0}} Q_{l_{k}}\right)=q_{l} q\left(\bigoplus_{k=1}^{m} P_{n_{0}} A_{i_{k}}\right)=q_{l}\left(\bigoplus_{k=1}^{m} P_{n_{0}} A_{i_{k}}\right) \subset \bigcap_{n \in N} P_{n} Q_{l},
$$

that is, $P_{n} Q_{l}=P_{n_{0}} Q_{l}$ for $n \geqslant n_{0}$. But by the Krull-Schmidt theorem $Q_{l}$ is isomorphic to one of the $A_{i_{k}}$ 's.

We have proved $P_{n} A_{j}=P_{n_{0}} A_{j}$ for $n \geqslant n_{0}$, provided that $A_{j}$ is isomorphic to an infinite number of $A_{i}$ 's, $i \in \mathbf{N}$. The same argument as above shows that for $j>n_{0}$ each $A_{j}$ which is not isomorphic to any $Q_{l}, l \in L^{\prime}$, also satisfies $P_{n} A_{j}=P_{n_{0}} A_{j}$ for $n>n_{0}$. Thus the number of $A_{j}$ 's violating this equation must be finite.

Now let $I$ be arbitrary. If our claim were false, there would be a family $\left(A_{i}\right)_{i \in I}$, a sequence $\left(i_{k}\right)_{k \in N}$ of pairwise different elements of $I$ and a strictly increasing sequence $\left(n_{k}\right)_{k \in N}$ of natural numbers so that $P_{n_{k}} A_{i_{k}} \supsetneqq P_{n_{k+1}} A_{i_{k}}$ for each $k$. Divide $I$ into pairwise disjoint subsets $I_{k}, k \in \mathrm{N}$, with $i_{k} \in I_{k}$ and define $M_{k}^{\prime}=\prod_{i \in I_{k}} M_{i}$. Then each $A_{i_{k}}$ is a direct summand of $M_{k}^{\prime}$, and our 
claim is also violated for the countable product $M=\Pi_{k \in N} M_{k}^{\prime}$ and the family $\left(A_{i_{k}}\right)_{k \in \mathrm{N}}$. But this we have already proved to be impossible.

REMARK. The same arguments as employed in the proof of Lemma 5 yield the following generalization of the implications $(1) \Rightarrow(3)$ and $(2) \Rightarrow(3)$ of Theorem A (the latter will be deduced directly from Lemma 5 in the sequel): If $\Pi_{i \in I} M_{i}$ possesses a direct-sum decomposition which complements direct summands, or if each product $\Pi_{i \in I} M_{i}$ with $\tilde{I} \subset I$ is a direct sum of modules with local endomorphism rings, then, for each descending chain $P_{1} \supset P_{2} \supset$ $P_{3} \supset \cdots$ of $p$-functors of $\operatorname{Mod} R$, there is a natural number $n_{0}$ so that $P_{n} M_{i}=P_{n_{0}} M_{i}$ for $n \geqslant n_{0}$ and almost all $i \in I$.

Proof of Theorem A. (1) $\Rightarrow(3)$. Let $M_{i}=M^{\mathbf{N}}$ for $i \in \mathbf{N}$. By hypothesis each $M_{i}$ is a direct sum of modules with local endomorphism rings, and so is $\Pi_{i \in \mathbf{N}} M_{i} \cong M^{\mathbf{N}}$. An application of Lemma 5 shows that $M^{\mathbf{N}}$ is $\Sigma$-a.c., hence $M$ has the same property. (2) $\Rightarrow$ (1) follows from [2, Proposition 12.10]. For the remaining implication $(3) \Rightarrow(2)$ note that $\Sigma$-algebraic compactness is inherited by all pure submodules of direct products of copies of $M$ (see the criterion preceding Lemma 4). Since moreover every $\Sigma$-a.c. module has an indecomposable decomposition [14, p. 1100], our claim is covered by Theorem $B$ following.

THEOREM B. Every $\Sigma$-algebraically compact module $M_{R}$ has the exchange property (i.e., $M^{\prime} \oplus N=\bigoplus_{i \in I} A_{i}$ with $M^{\prime} \cong M$ implies the existence of submodules $C_{i}$ of $A_{i}$ such that $M^{\prime} \oplus N=M^{\prime} \oplus \bigoplus_{i \in I} C_{i}$ ).

Proof. Suppose that $A=M^{\prime} \oplus N=\bigoplus_{i \in I} A_{i}$ with $M^{\prime} \cong M$. Since $M^{\prime}$ is again $\Sigma$-a.c., we may assume $M^{\prime}=M$. By Zorn's lemma there is a maximal submodule $C=\bigoplus_{i \in I} C_{i}$ of $A$ with $C_{i} \subset A_{i}$ for all $i$, so that $M \cap C=0$ and the canonical monomorphism $M \rightarrow A / C \cong \bigoplus_{i \in I} A_{i} / C_{i}$ is pure. The canonical image $\bar{M}$ of $M$ in $\bar{A}=A / C$ being also $\Sigma$-a.c., we have $\bar{A}=\bar{M} \oplus X$. We wish to show $X=0$. In order to simplify the notation, we replace $\bar{A}$ by $A$ and $\bar{M}$ by $M$, i.e. we assume the following situation: $A=M \oplus X=\bigoplus_{i \in I} A_{i}$ with $M \Sigma$-a.c. so that, for $0 \neq A_{i}^{\prime} \subset A_{i}$ with $M \cap A_{i}^{\prime}=0$, the sum $M \oplus A_{i}^{\prime}$ is not a direct summand of $A$ (the latter is a consequence of the choice of $C$ ).

First, we recognize that in this setting $A$ is $\Sigma$-a.c.: $M$ has the finite exchange property by [15, p. 87(2)], so, fixing $h \in I$, we have $A=M \oplus A_{h}^{\prime} \oplus A^{\prime}$ with $A_{h}^{\prime} \subset A_{h}$ and $A^{\prime} \subset \bigoplus_{i \in I \backslash h\}} A_{i}$. It follows that $A_{h}^{\prime}=0$, hence $A_{h}$ is isomorphic to a direct summand of $M$, and our first claim is established. In particular, all of the $A_{i}$ 's, as well as $X$, can be decomposed into submodules with local endomorphism rings, say $A_{i}=\bigoplus_{l \in L_{i}} A_{i l}$ and $X=\bigoplus_{j \in J} X_{j}$, see $[14$, p. 1100]. If $X$ were nonzero, i.e. $J$ nonempty, say $k \in J$, then the module $M \oplus \bigoplus_{j \in J \backslash k\}} X_{j}$ would be a maximal direct summand of $A$. Since by Azumaya's theorem (see, e.g., [2, Theorem 12.6]) the decomposition $A=$ $\bigoplus_{i \in I ; l \in L_{i}} A_{i l}$ complements maximal direct summands, we would infer 


$$
A=\left(M \oplus \underset{j \in J \backslash\{k\}}{\bigoplus} X_{j}\right) \oplus A_{i l}
$$

for some $i$ and $l$, which is incompatible with the initial situation. Thus, we conclude $X=0$.

Given a $\Sigma$-algebraically compact module $M$, write $M=\bigoplus_{i \in I} M_{i}$ with $\operatorname{End}_{R}\left(M_{i}\right)$ local (by [14, p. 1100] such a decomposition always exists). According to [12, Proposition 2], each finite sum $M^{\prime}=\bigoplus_{i \in I^{\prime}} M_{i}$ has the cancellation property (i.e., $A \oplus M^{\prime} \cong B \oplus M^{\prime}$ implies $A \cong B$ ), but of course this fails for infinite sums. Before being allowed to cancel $M$ in a relation $A \oplus M \simeq B$ $\bigoplus M$, we must clear $A$ and $B$ of direct summands of $M$. More precisely, the following is true:

COROLlaRY 6. Let $M$ be $\Sigma$-algebraically compact, $M=\bigoplus_{i \in I} M_{i}$ an indecomposable decompositon. Then every $R$-module $A$ can be uniquely (up to isomorphism) decomposed in the form $A=A_{1} \oplus A_{2}$ where $A_{1}$ is isomorphic to a direct sum of $M_{i}$ 's and $A_{2}$ and $M$ have no common nonzero direct summand. In particular, $A \oplus M \cong B \oplus M$ with $A=A_{1} \oplus A_{2}$ and $B=B_{1} \oplus B_{2}$ as above implies $A_{2} \cong B_{2}$ and $A_{1} \oplus M \cong B_{1} \oplus M$.

Proof. Existence: By Zorn's lemma there is a maximal family $\left(C_{j}\right)_{j \in J}$ of submodules of $A$ with the following properties:

(1) each $C_{j}$ is isomorphic to some $M_{i}$;

(2) the sum $\sum_{j \in J} C_{j}$ is direct;

(3) each finite subsum $\Sigma_{j \in J}, C_{j}$ is a direct summand of $A$.

Since $A_{1}=\bigoplus_{j \in J} C_{j}$ is $\Sigma$-a.c. and a pure submodule of $A$, we have $A=A_{1} \oplus A_{2}$ for some submodule $A_{2}$ of $A$. The maximality of $\left(C_{j}\right)_{j \in J}$ guarantees that $A_{2}$ contains no direct summand isomorphic to one of the $M_{i}$ 's. It follows that $M$ and $A_{2}$ have no nonzero isomorphic direct summand at all, because direct summands of $M$ are in turn $\Sigma$-a.c. and hence direct sums of indecomposable modules (necessarily isomorphic to certain $M_{i}$ 's).

Uniqueness: Suppose $A=A_{1} \oplus A_{2}=D_{1} \oplus D_{2}$ are two decompositions of the considered type. By investing the exchange property of $A_{1}$ (Theorem B), we obtain an equation $A=A_{1} \oplus D_{1}^{\prime} \oplus D_{2}^{\prime}$ with $D_{i}^{\prime} \subset D_{i}$ for $i=1,2$, say $D_{i}=D_{i}^{\prime} \oplus D_{i}^{\prime \prime}$. From the property of $A_{2}$ we infer $D_{1}^{\prime}=0$, and, in view of $A_{1} \simeq D_{1} \oplus D_{2}^{\prime \prime}$, we obtain further $D_{2}^{\prime \prime}=0$. This means $A_{1} \cong D_{1}$ and $A=$ $A_{1} \oplus D_{2}$. But the latter shows that also $A_{2} \cong D_{2}$.

We restate Theorem B (resp., Corollary 6) for a special class of $\Sigma$-a.c. modules.

COROllaRY 7. Let $\left(M_{i}\right)_{i \in I}$ be a family of $R$-modules containing only finitely many isomorphism types and suppose that the modules $M_{i}$ are artinian over their endomorphism rings. Then every direct summand $M$ of the direct sum or the direct product of the $M_{i}$ 's has the exchange property and the cancellation property of Corollary 7. Moreover, $M$ has a decomposition that complements direct summands. 
For $\boldsymbol{R}$ commutative, this extends [2, Corollary 29.6], for $R$ a Dedekind domain we rediscover classical theorems on bounded $R$-modules. Moreover, we infer that, for $R$ right $\Sigma$-algebraically compact (e.g., for $R$ left artinian), every right $R$-module $A$ has (uniquely up to isomorphism) a decomposition $A=A_{1} \oplus A_{2}$ where $A_{1}$ is projective and $A_{2}$ has no projective direct summand; in particular, given an isomorphism of $R$-modules $A \oplus M \simeq B \oplus$ $M$, where $M$ is projective and $A, B$ have no nonzero projective direct summands, $M$ may be cancelled.

\section{REFERENCES}

1. F. W. Anderson and K. R. Fuller, Modules with decompositions that complement direct summands, J. Algebra 22 (1972), 242-253.

2. __ Rings and categories of modules, Springer-Verlag, Berlin, 1973.

3. M. Auslander, Notes on representation theory of artin algebras, Brandeis Univ., Waltham, Mass., 1972.

4. S. U. Chase, Direct products of modules, Trans. Amer. Math. Soc. 97 (1960), 457-473.

5. __ On direct sums and products of modules, Pacific J. Math. 12 (1962), 847-854.

6. C. Faith and E. A. Walker, Direct-sum representations of injective modules, J. Algebra 5 (1967), 203-221.

7. K. R. Fuller and I. Reiten, Note on rings of finite representation type and decomposition of modules, Proc. Amer. Math. Soc. 50 (1975), 92-94.

8. K. R. Fuller, On rings whose left modules are direct sums of finitely generated modules, Proc. Amer. Math. Soc. 54 (1976), 39-44.

9. L. Gruson and C. U. Jensen, Deux applications de la notion de L-dimension, C. R. Acad. Sci. Paris Sér. A 282 (1976), 23-24.

10. C. M. Ringel and H. Tachikawa, QF-3 rings, J. Reine Angew. Math. 272 (1975), 49-72.

11. R. B. Warfield, Jr., Purity and algebraic compactness for modules, Pacific J. Math. 28 (1969), 699-719.

12. , A Krull-Schmidt theorem for infinite sums of modules, Proc. Amer. Math. Soc. 22 (1969), 460-465.

13. __ Rings whose modules have nice decompositions, Math. Z. 125 (1972), 187-192.

14. W. Zimmermann, Rein injektive direkte Summen von Moduln, Comm. Algebra 5 (1977), 1083-1117.

15. B. Zimmermann-Huisgen and W. Zimmermann, Algebraically compact rings and modules, Math. Z. 161 (1978), 81-93.

Institut für Mathematix der Technischen Universttät MǗnChin, ARCisstrasse 21, 8 Munich 2, Federal Republic of Germany 